\title{
Endogenous Oscillations in a Discrete Dynamic Model with Inventory
}

\author{
AKIO MATSUMOTO* \\ Niigata University, Department of Economics, 8050, 2-No-cho, Ikarashi, Niigata, 950-21, Japan
}

( Received 15 June 1996)

\begin{abstract}
Introducing the producer's intertemporal optimizing behavior, we extend the Eckalbar Disequilibrium Macro-Model (1985) and reconsider the dynamic features of the modified model. We concern ourselves with the existence of inventory cycles when the expectations are formed adaptively. The endogenous inventory cycle is detected using the Hopf bifurcation theorem in which a bifurcation parameter is an adaptive coefficient. It is also demonstrated that the generated cycle is subcritical.
\end{abstract}

Keywords: Hopf bifurcation, Limit cycle, Stability index

\section{INTRODUCTION}

This study analyzes non-linear dynamics of a simple disequilibrium macro-model with inventories. The main purpose is to investigate what role the profit-maximizing firm plays to generate cyclic inventory dynamics. The model has linear demand and non-linear supply, the latter of which is an outcome of intertemporal profit maximization by the firm. The Hopf bifurcation theorem is used to demonstrate the existence of endogenous inventory cycles. Further, an example is presented to show that the generated cycles are subcritical when the production cost function is linear.
Inventory-theoretic macro-models are developed in the framework of disequilibrium economics. Several stability or unstability results have been established. ${ }^{1}$ This study extends Eckalbar (1985) by introducing the optimal behavior of the profit maximizing firm. Eckalbar constructs a continuous-time macro-model in which expectations on sales are adaptively adjusted and establishes the existence of limit cycles, applying the Poincaré-Bendixson theorem. The dynamic system employed is non-linear, but sources of nonlinearity are exogenously determined. That is, lower and upper bounds of variables such as fullemployment output and non-negative employment are exogenously introduced and work to

\footnotetext{
*Tel.: +81/(0)25-262-6551. Fax: +81/(0) 25-263-3262. E-mail: eakio@ @le.niigata-u.ac.jp.

'See Honkapohja and Ito (1980), Simonovits (1982), Eckalbar (1985) and Franke and Lux (1993).
} 
prevent the unstable behavior from expanding globally. In particular, these exogenous variables define switching lines to divide the phase space into subregions and make the system a sort of dynamical hybrid. Thus, in one region divided by the switching lines, one unstable subsystem governs the dynamic variables and drives these away from the equilibrium point. In another region, another stable subsystem governs the same variables and drives these back to the region in which the equilibrium point exists. The dynamic variables oscillate back and forth in these regions. When the stabilizing force is balanced against the unstabilizing force, the cyclic dynamics can emerge in such models. Coexistence of opposite-directed dynamic forces is due to the exogenous factors.

There are some directions in which the Eckalbar linear model is extended. In the first half of their study, Poston et al. (1992) refine on the Eckalbar model and clarify the conditions for sustained oscillation. Zhang (1989) and Poston et al. (1992) (in the latter half) generalize the Eckalbar's piecewise linear model with the purpose of elucidating endogenous oscillations independent of the exogenous factors. Zhang introduces a non-linear adjustment function of the desired inventory while Poston et al. introduce a non-linear inventory investment function. In doing so, they replace the exogenously determined switching dynamic system of the Eckalbar model with the endogenously determined non-linear dynamic systems and demonstrate the existences of endogenous inventory cycles, the existences of which a Hopf bifurcation theorem is applied to establish. In those studies, however, non-linearity, which is sufficiently strong to bring out cyclic dynamics, has been assumed more or less directly on the dynamical system or on the economic behavior of a particular body of agents. It has been known that the dynamic model endowed with a sufficient non-linearity can generate complex dynamics. Little is known about a source of such non-linearity. To go one step further, we construct a micro-foundation of the Eckalbar macro-model and shed light on its non-linear structure in which the optimal behavior of the profit maximizing firm plays an important role for generations of cyclic dynamics.

The fundamental characteristics of our model are similar to those of the Zhang model as well as the Poston model and thus those of the Eckalbar model. But there are many deviations from these models. First, our model is cast in discrete time, whereas their models are in continuous time. It is worthwhile to consider a discrete version of the Eckalbar model because the dynamics generated by a discrete-time system is significantly different from the dynamics by a continuous-time system. ${ }^{2}$ Second, we derive choice-theoretically the producer's behavioral functions based on the intertemporal profit maximization. This provides a microeconomic foundation of the supply side of the model. In the models of Zhang and Poston et al., the non-linearity of the desired stock adjustment function or the inventory investment function is, as mentioned above, assumed directly on the producer's behavior. Third, although we also apply a discrete-time Hopf bifurcation theorem to show endogenous oscillations, a bifurcation parameter in our model is a coefficient of the adaptive expectations on sales, whereas it is the marginal propensity to consume in the Zhang model. In this study, it is demonstrated that the endogenous inventory cycle can emerge when a set of adaptive coefficient and inventory-expectation ratio crosses critical values for which the characteristic roots of the dynamical system become complex.

The paper is organized as follows. Section 2 constructs a basic model based on individual's optimizing behavior. Section 3 considers inventory dynamics with adaptive expectations. Section 4 makes concluding remarks.

\footnotetext{
${ }^{2}$ Dana-Malgrange (1984) simulates the Kaldor continuous growth model with a discrete-time basis and shows different qualitative properties.
} 


\section{THE BASIC MODEL}

This section recapitulates the fundamental structure of Eckalbar's model and introduces the producer's dynamic optimizing behavior. A model has two traders and three commodities. Three commodities are aggregate consumption goods, labor, and money. The goods are assumed to be storable. Two trades are a consumer and a producer. ${ }^{3}$ Exchange takes place through money so that there are two markets: the consumption goods market and the labor market. The price of the consumption goods, $p$, and the wage rate, $w$, are exogenously fixed. These prices not being equilibrium one, demand is not necessarily equal to supply in each market.

For the sake of simplicity, we make two assumptions to determine the actual quantity traded in each market. First, the markets are assumed to operate sequentially so that the traders enter the labor market and then the goods market. Second, actual transaction in a disequilibrium market is assumed to be determined by the minimum of supply and demand (i.e., the "min-rule" or "short-side" rule of disequilibrium theory). Accordingly, the traders find a difference between what they expect to trade at the start of a period and what they actually realize at the end. The difference not only determines the initial level of buffer stocks in the following period but also affects the revision of expectations of the market state on which the traders base their economic decisions. Consequently, the macrodynamics evolves.

We divide the remaining part of this section into three parts. In the first part, we describe the consumer's behavior. Since our emphasis of this study is placed on how the producer's optimal behavior affects the macro-dynamics, we specify the consumer's behavior as simple as possible. In the second, we describe the intertemporal optimal behavior of the firm. In the third, we examine determination of output, employment and inventory accumulation.

\subsection{Consumer}

We make a behavioral specification on the representative consumer in this subsection. For the sake of brevity, we do not formulate the utility maximization problem of the consumer but assume the inelastic supply of labor, $N{ }^{4}$ and the linear - Keynesian-type - expenditure function. Since the labor market operates first, the consumer knows if he is fully employed or not when he enters the goods market. Having the actual quantity traded in the labor market, $L$, the consumer makes a choice of consumption demand, $S$, by

$$
S(L)=c_{0}+c L
$$

where $c_{0}>0$ is the demand for the goods in the case of unemployment and $c$ is the marginal propensity to demand with respect to employment.

\subsection{Firm}

We describe the producer's intertemporal profit maximization behavior in this subsection. $y$ is the quantity of the consumption goods produced by using employed labor, $L$, with the conventional production function, $y=F(L) . h$ is an initial stock of inventory at the start of a decision period and $h_{+1}$ the inventory carried-over to the following period. Hence the inventory accumulation equation is

$$
h_{+1}=h+y-S \text {. }
$$

$s$ is an expectation on sales that the producer forms before entering the goods market. We assume that

\footnotetext{
${ }^{3}$ We suppose that our economy is composed of a fixed number of identical producers and of identical consumers. Hence, the analysis focuses on the behavior of the representative producer and the representative consumer, each of whom is taken to reflect the corresponding aggregate behavior.

${ }^{4}$ The same assumption is made in Honkapohja and Ito (1980), Simonovits (1982), and Eckalbar (1985).
} 
the producer has the desired level of inventory, denoted by $\tilde{h}$, to be a fixed ratio of expected sales to stock:

$$
\tilde{h}=\beta s, \quad \text { where } \beta>0 .
$$

We make the fixed ratio assumption in order to clarify the firm's contributions to persistent cyclical behavior of inventory. ${ }^{5}$ As is seen later in the dynamic analysis, the firm's profit maximizing behavior leads to a non-linear dynamic system even under the fixed-ratio assumption.

The producer incurs two types of cost: the cost of producing output and the cost of holding inventory. We denote by $C(y)$ the cost associated with producing $y$. Labor being the only input for the production function, it is the labor cost. That is, $C(y)=w F^{-1}(y)$, where $F^{-1}(y)$ is an inverse of the production function and denotes the quantity of labor necessary to produce output, $y$. We assume that the cost of holding inventory is associated with a deviation of an actual level of inventory from the desired level of inventory, and denote it by $H(h-\tilde{h}) . V\left(h_{+1}\right)$ is the maximum of the expected profit that the producer achieves by employing the best policy from the next period and onwards. ${ }^{6}$ It accounts for the discount factor. We make the following assumptions on these functions:

\section{ASSUMPTION 1}

(1) $F^{\prime}(L)>0$ and $F^{\prime \prime}(L)<0$,

(2) $H^{\prime}\left(h_{+1}-\tilde{h}\right) \lesseqgtr 0$ for $h \lesseqgtr \tilde{h}, \lim _{h \rightarrow 0} H^{\prime}\left(h_{+1}-\right.$ $\tilde{h})=-\infty, \lim _{h \rightarrow \infty} H^{\prime}\left(h_{+1}-\tilde{h}\right)=\infty$, and $H^{\prime \prime}$ $\left(h_{+1}-\tilde{h}\right)>0$

(3) $V^{\prime}(h)>0$ and $V^{\prime \prime}(h)<0$.

Assumption 1(1) states that the marginal productivity of labor is positive and further employ- ment brings about further but smaller production increases. As a result of this assumption, the marginal cost of production is positive and increasing. Assumption 1(2) states that as an actual level of inventories, $h$, deviates from the desired level of inventory, $\tilde{h}$, the cost of holding inventory increases due to the loss of the goodwill for negative deviation (i.e., $h<\tilde{h}$ ) and due to the increase of the storage cost for positive deviation (i.e., $h>\tilde{h}$ ). Further, the convexity is assumed. Assumption 1(3) describes that the imputed real values increases at a decreasing rate as inventories increase. ${ }^{7}$

We solve the firm's optimization problem. The firm chooses production and inventory carriedover so as to maximize the expected profit,

$$
p s-C(y)-H\left(h_{+1}-\tilde{h}\right)+V\left(h_{+1}\right)
$$

subject to the non-negative constrains on decision variables,

$$
y \geq 0 \quad \text { and } \quad h_{+1}=h+y-s \geq 0,
$$

where the firm takes only intended change of inventory into account when choosing the optimal plan. The Lagrangian of the profit maximization problem is

$$
\begin{aligned}
\mathcal{L}= & p s-C(y)-H\left(h_{+1}-\tilde{h}\right)+V\left(h_{+1}\right) \\
& +\lambda\{y-\max (0, s-h)\} .
\end{aligned}
$$

Differentiating Eq. (6) with respect to $y$ yields the first-order condition for the optimal production,

$$
g^{\prime}\left(h_{+1}\right)-C^{\prime}(y)+\lambda=0,
$$

where $g^{\prime}\left(h_{+1}\right)=V^{\prime}\left(h_{+1}\right)-H^{\prime}\left(h_{+1}-\tilde{h}\right)$ is the marginal future revenue subtracting the marginal

\footnotetext{
${ }^{5}$ Eckalbar makes the fixed ratio assumption with three reasons: (1) it is easy to work with; (2) it captures the spirit of the micro-level stocks literature; (3) it is in line with the fact. As already has been stated, Zhang (1989) replaces the fixed-ratio adjustment with the non-linear adjustment function and obtains persistent cyclical behavior.

${ }^{6} \mathrm{We}$ can take a more rigorous approach to the multiperiod optimizing problem of the firm. We, however, do not do so for two reasons. First, we avoid to solve the complicated mathematical problem. Second, the gist of this paper is not to rigorously study the details of an inventory-holding firm but rather to reveal the role of a profit maximizing firm and the dynamics of the model. We use the simpler approach to inventory-holding behavior in order to highlight the purpose of the paper. Our approach approximates it and captures its essential features.

${ }^{7}$ There are some studies in a literature of optimal inventory theory that can be used to entail Assumption 1(3).
} 
cost for carrying inventories to the future period, and where $\lambda$ is a Lagrange multiplier associated with the non-negative constraint. An optimal condition for maximizing profit, (7), indicates that the cost of producing one additional unit today and storing it until tomorrow is not less than the revenue gained by selling one unit out of the inventory stock tomorrow. The optimal production depends on the relative magnitude among the initial level of inventory, $h$, the expectation on sales, $s$, and a level of inventory, denoted by $h_{0}(s)$, that equates the marginal revenue to the marginal cost of holding inventory. ${ }^{8}$ In particular, if $h-s>h_{0}(s)$ holds, we have $g^{\prime}\left(h_{+1}\right)<0$ and $C^{\prime}\left(h_{+1}-(h-s)\right)>0$ for any $h_{+1}>h-s$. In this case, the first-order condition, (7), leads to no production. That is, if the initial level of inventory is large enough, the cost of holding inventory is over the expected return so that the producer does not produce at all but liquidates stocks of inventory to meet demand for the consumption goods. On the other hand, if $h-s<$ $h_{0}(s)$ holds, the optimal production, denoted by $y^{*}(s, h)$, satisfies the following condition:

$$
\begin{aligned}
C^{\prime}\left(y^{*}(s, h)\right)= & V^{\prime}\left(h+y^{*}(s, h)-s\right) \\
& -H^{\prime}\left(h+y^{*}(s, h)-s-\tilde{h}\right) .
\end{aligned}
$$

Changes in an initial level of inventory and of expectation on sales alter the optimal level of production. A standard comparative statics exercise for the optimal production yields the following effects on the equilibrium production:

$$
\begin{gathered}
-1<\frac{\partial y^{*}}{\partial h}=-\frac{H^{\prime \prime}-V^{\prime \prime}}{C^{\prime \prime}+H^{\prime \prime}-V^{\prime \prime}}<0, \\
0<\frac{\partial y^{*}}{\partial s}=\frac{\beta H^{\prime \prime}+H^{\prime \prime}-V^{\prime \prime}}{C^{\prime \prime}+H^{\prime \prime}-V^{\prime \prime}} \geq 1 \quad \text { as } \beta \geq \frac{C^{\prime \prime}}{H^{\prime \prime}} .
\end{gathered}
$$

Inequality conditions on Eq. (9) indicate that an increase in initial level of inventory reduces production but not the entire amount of the increase. The remaining amount is met with decreases in the optimal inventory carried-over. ${ }^{9}$ The change in the expected sales shifts the $g^{\prime}\left(h_{+1}\right)$ curve and the $C^{\prime}(y)$ curve, both of which affect the optimal production. If the shift of the $g^{\prime}\left(h_{+1}\right)$ curve dominates the shift of the $C^{\prime}(y)$ curve (i.e., $\left.\beta H^{\prime \prime}>C^{\prime \prime}\right)$, changes in the optimal production is greater than the change in the expected sales, and vice versa. This is what the second inequalities in Eq. (10) indicates.

These considerations imply that the demand for labor has two phases:

$$
L^{\mathrm{d}}(s, h)=\max \left\{0, F^{-1}\left(y^{*}(s, h)\right)\right\} .
$$

Partial derivatives of positive demand for labor are

$$
\begin{aligned}
& \frac{\partial L^{\mathrm{d}}}{\partial h}=\frac{1}{F^{\prime}\left(y^{*}\right)}-\frac{\partial y^{*}}{\partial s}>0 \quad \text { and } \\
& \frac{\partial L^{\mathrm{d}}}{\partial s}=\frac{1}{F^{\prime}\left(y^{*}\right)} \frac{\partial y^{*}}{\partial s}>0 .
\end{aligned}
$$

\subsection{Determination of Actual Transaction}

We consider determination of actual transactions in the labor market and the goods market. We restrict our analysis to a "Keynesian" state. That is to say, the consumer achieves his desired transaction in the goods market and cannot in the labor market while the producer can achieve his transaction in the labor market and cannot in the goods market. In order to highlight the endogenous non-linearity of the model, we assume that the exogenous amount of labor supply, $N$, is not a binding constraint in the labor market.

The model functions as follows. At the start of a period, the producer holds an initial stock of inventory, $h$, and forms a subjective expectation

\footnotetext{
${ }^{8}$ Assumption 1(2) and (3) imply that $g^{\prime}\left(h_{+1}\right)>0$ for a small enough level of $h_{+1}$, and $g^{\prime}\left(h_{+1}\right)<0$ for a large enough level of $h_{+1}$, and that $g^{\prime \prime}\left(h_{+1}\right)=V^{\prime \prime}\left(h_{+1}\right)-H^{\prime \prime}\left(h_{+1}-\tilde{h}\right)<0$. Thus there is a level of inventory, $h_{0}(s)$, such that $g^{\prime}\left(h_{0}(s)\right)=0$ or $V^{\prime}\left(h_{0}(s)\right)=H^{\prime}\left(h_{0}(s)-\tilde{h}\right)$.

${ }^{9}$ We can see this by differentiating the intended inventory accumulation equation in (5) where $y$ is replaced with $y^{*}(s, h)$ and $h$ with the optimal inventory carried-over, $h_{+1}^{*}$ (i.e., $\partial h_{+1}^{*} / \partial h+\left(-\partial y^{*} / \partial h\right)=1$ ).
} 
on current sales, $s$. Following the analysis above, the producer determines his desired demand for labor $L^{\mathrm{d}}(s, h)$ while the consumers offers a fixed quantity of labor supply, $N$. The consumer and the producer meet first in the labor market in which there exists excess supply. According to the min-rule, the demand side of the labor market determines the actual quantity of labor employed, $L$ :

$$
L=\min \left\{N, L^{\mathrm{d}}(s, h)\right\}=L^{\mathrm{d}}(s, h) .
$$

After the labor market closes, the consumer chooses his demands for the consumption goods, $S(L)$. Actual employment also determines the current production for output, $F(L)$. The starting stock of inventory is a sum of the current production and initial inventory, $h+F(L)$. This is the supply of the goods that we denote by $Y^{\mathrm{s}}(L)$. The producer is assumed to hold enough amount of inventory so that the consumer always realize his desired demand for the consumption goods. Thus the actual sales, $Y$, is the demand for the consumption goods:

$$
Y=\min \left\{Y^{\mathrm{s}}(L), S(L)\right\}=S(L) .
$$

At the end of the period, transactions complete and the economy is in a temporary equilibrium state in which the sum of actual purchases equals the sum of actual sales. A difference between the demand for the goods and output produced determines an actual level of inventory carried over to the next period. Moreover, the producer recognizes a difference between the expectation on sales and the actual demand. Consequently, the producer adjusts his expectations on sales in the following period. Hence the inventory accumulation and the expectation revision can be sources of dynamics of the model.

Equation (2) governs the inventory accumulation process. In order to describe dynamics of the model, we need to specify how the expectation is revised from one period to the next. In the following section, we assume a formation of adaptive expectations. That is, the producer adaptively adjusts his expectation according to a difference between demand for the consumption goods and current level of expectation,

$$
s_{+1}=s+\alpha(S-s) \text {, }
$$

where $\alpha$ is the adjustment coefficient and $s_{+1}$ denotes the expectation one period ahead.

\section{INVENTORY DYNAMICS WITH ADAPTIVE EXPECTATION}

In this section we demonstrate that endogenous inventory cycles appear when the speed of expectation adjustment is varied. ${ }^{10}$ The dynamic system that governs the expectation on sales, $s_{t}$, and the level of inventory, $h_{t}$, is

$$
\begin{aligned}
& s_{t+1}=s_{t}+\alpha\left(S\left(F^{-1}\left(y^{*}\left(s_{t}, h_{t}\right)\right)\right)-s_{t}\right), \\
& h_{t+1}=h_{t}+y^{*}\left(s_{t}, h_{t}\right)-S\left(F^{-1}\left(y^{*}\left(s_{t}, h_{t}\right)\right)\right),
\end{aligned}
$$

where the first is the expectation revised equation and the second is the inventory accumulation equation. In the following, we illustrate the dynamic behavior of the model in phase diagrams as a first-order approximation and then analyze it mathematically. Before proceeding, we define an equilibrium state of the model that is a fixed point of the dynamic system, (16).

DEFINITION 1 An equilibrium state of the macromodel with adaptive expectations is a pair of expectation and inventory, $\left(s^{*}, h^{*}\right)$, such that $y^{*}\left(s^{*}, h^{*}\right)$ $=S\left(F^{-1}\left(y^{*}\left(s^{*}, h^{*}\right)\right)\right)$ and $S\left(F^{-1}\left(y^{*}\left(s^{*}, h^{*}\right)\right)\right)=s^{*}$.

\subsection{Graphical Analysis}

To make a graphical analysis of the behavior of inventory, $h_{t}$, we find the locus of $\left(s_{t}, h_{t}\right)$ points along which the level of inventory is constant.

\footnotetext{
${ }^{10} \mathrm{~A}$ time subscript, $t$, is attached to time-dependent variables hereon.
} 
This locus, which we call the constant inventory locus, must satisfy

$$
y^{*}\left(s_{t}, h_{t}\right)=S\left(F^{-1}\left(y^{*}\left(s_{t}, h_{t}\right)\right)\right) .
$$

To put it another way, output produced equals the quantity demand for $\left(s_{t}, h_{t}\right)$ on the constant inventory locus. Such an equality holds at a point where the production curve, $F(L)$, crosses the demand curve, $S(L)$. Both curves are increasing at non-increasing rates with respect to $L$. There will be no intersection, one, or two depending on exogenously determined parameters like prices, wage, autonomous demand, consumer's characteristics, properties of the production function, etc. We make the following assumption to ensure the intersections.

Assumption 2 The $F(L)$ curve intersects the $S(L)$ curve twice.

Since $F(0)=0<S(0)$, Assumption 2 implies that the production curve crosses the demand curve from below and then from left as $L$ increases from zero to infinity. We denote the first intersection by $\left(y_{1}, L_{1}\right)$ and the second intersection by $\left(y_{2}, L_{2}\right)$ where $y_{i}$ is output produced with $L_{i}$ (i.e. $y_{i}=F\left(L_{i}\right)$ for $i=1,2$ ). At these points, the following inequality conditions hold:

$$
F^{\prime}(L)>S^{\prime}(L) \text { for } L=L_{1}
$$

and $\quad F^{\prime}(L)<S^{\prime}(L)$ for $L=L_{2}$.

As a result of Assumption 2, there are two constant inventory loci: one corresponds to the lower production, $y^{*}\left(s_{t}, h_{t}\right)=y_{1}$, and the other to the higher production, $y^{*}\left(s_{t}, h_{t}\right)=y_{2}$. The constant inventory locus crosses the $h_{t}$ axis for $h_{t}$ that satisfies $y^{*}\left(0, h_{t}\right)=y_{i} \quad(i=1,2)$. This intercept is positive or negative according to whether $y_{i}$ is less or greater than the optimal level of production for $s=h=0 .^{11}$
Determining the slope of the locus by differentiating Eq. (17), we find from (9) and (10) that it has a positive slope in the $\left(s_{t}, h_{t}\right)$ plane:

$$
0<\left.\frac{\partial h}{\partial s}\right|_{h_{t+1}=h_{t}}=-\frac{\partial y^{*} / \partial s}{\partial y^{*} / \partial h} .
$$

We differentiate the second equation in dynamic system (16) and then transform the resultant equation into

$$
\frac{\partial\left(h_{t+1}-h_{t}\right)}{\partial h_{t}}=\left(1-\frac{S^{\prime}}{F^{\prime}}\right) \frac{\partial y^{*}}{\partial h_{t}} .
$$

Since the second factor, $\partial y^{*} / \partial h_{t}$, is negative by Eq. (9), the sign of Eq. (20) depends upon the relative magnitude of the marginal product, $F^{\prime}$, and the marginal propensity to demand with respect to employment, $S^{\prime}$. By Assumption 2, we have $F^{\prime}(L)>S^{\prime}(L)$ for $L=L_{1}$ and $F^{\prime}(L)<S^{\prime}(L)$ for $L=L_{2}$. Thus $h_{t+1}<h_{t}$ if and only if $\left(s_{t}, h_{t}\right)$ lies above the locus producing $y_{1}$, while $h_{t+1}>h_{t}$ if and only if $\left(s_{t}, h_{t}\right)$ lies above the locus producing $y_{2}$.

We can also determine a locus of $\left(s_{t}, h_{t}\right)$ along which the expectation on sales is constant and will call it the constant expectation locus. Following the first difference equation of the dynamic system, (16), the locus satisfies

$$
S\left(F^{-1}\left(y^{*}\left(s_{t}, h_{t}\right)\right)\right)=s_{t},
$$

which means that the expectation on sales is realized. It is verified that this locus intersects the $y^{*}\left(s_{t}, h_{t}\right)=0$ locus at a point $\left(s^{0}, h^{0}\right)$ where $s^{0}=$ $S(0)$ and $h^{0}$ satisfies $y^{*}\left(s^{0}, h^{0}\right)=0 .{ }^{12}$

By totally differentiating Eq. (21), we obtain the slope of the constant expectation locus:

$$
\left.\frac{\partial h}{\partial s}\right|_{s_{t+1}=s_{t}}=\frac{\partial y^{*} / \partial s}{S^{\prime} \partial y^{*} / \partial h}\left(\frac{F^{\prime}}{\partial y^{*} / \partial s}-S^{\prime}\right)
$$

\footnotetext{
${ }^{11}$ Suppose that $y_{0}$ satisfies the optimal condition, $C^{\prime}\left(y_{0}\right)=V^{\prime}\left(y_{0}\right)-S^{\prime}\left(y_{0}\right) \cdot y^{*}\left(s_{t}, h_{t}\right)=y_{0}$ is a locus of $\left(s_{t}, h_{t}\right)$ starting at the origin, $(0,0)$ in the $\left(s_{t}, h_{t}\right)$ plane. In Fig. 1 , we assume $y_{1}<y_{0}<y_{2}$.

${ }^{12}$ For $\left(s^{0}, h^{0}\right)$, the demand for the goods is $S\left(F^{-1}\left(y^{*}\left(s^{0}, h^{0}\right)\right)\right)=S(0)=s^{0}$. Thus the constant expectation locus passes through the point $\left(s^{0}, h^{0}\right)$. For $\left(s_{t}, h_{t}\right)$ over the $y^{*}\left(s_{t}, h_{t}\right)=0$ locus, zero production takes place. Hence the constant expectation locus is $s^{0}=s_{t}$.
} 


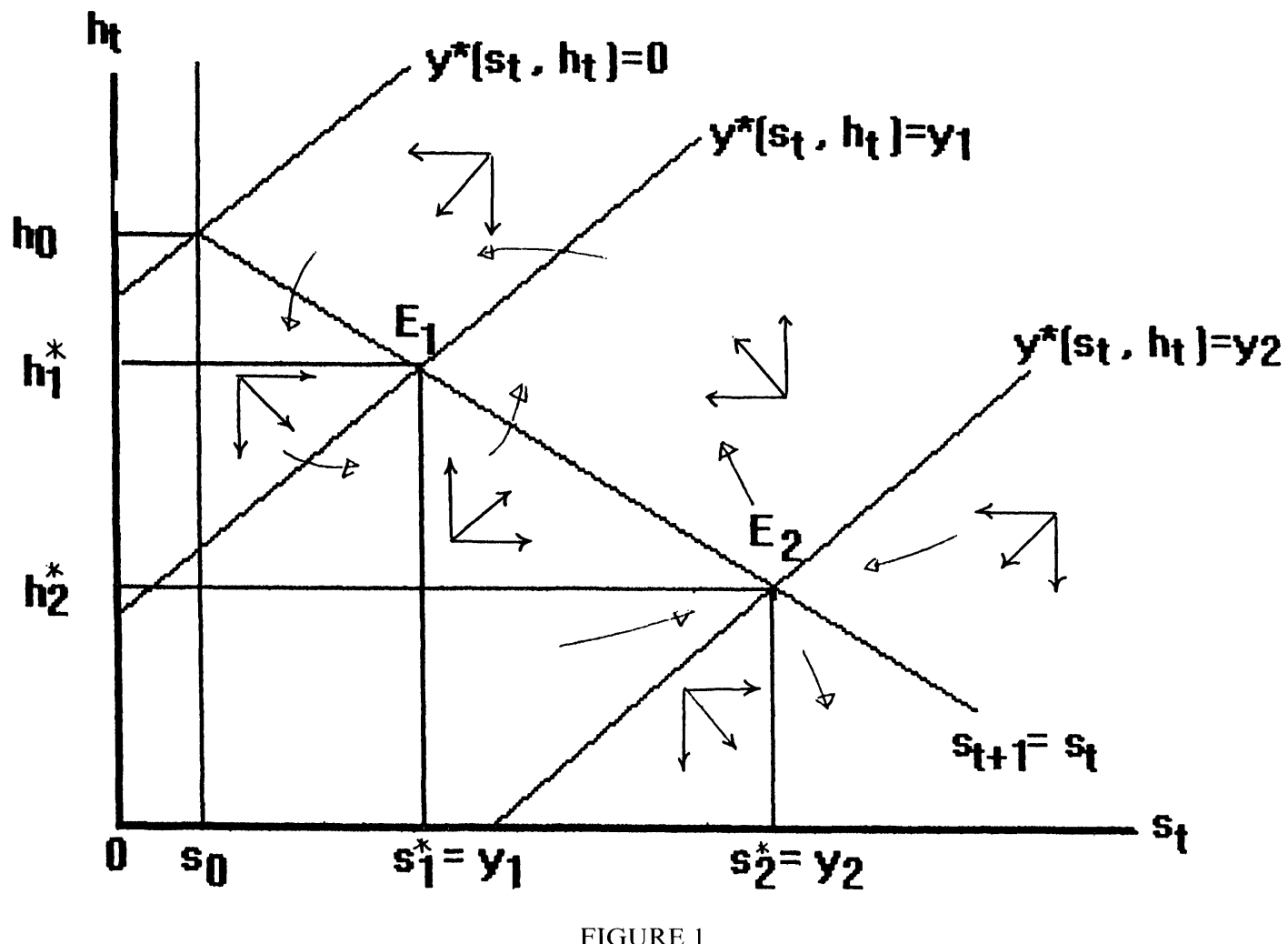

where the first factor is negative but the sign of the second factor is ambiguous. $F^{\prime} /\left(\partial y^{*} / \partial s\right)$ is a reciprocal of the second equation in Eq. (12). It is a slope of the $L=L^{\mathrm{d}}\left(s_{t}, h_{t}\right)$ curve that, for each level of employment, $L$, measures what the expectation on sales would have to be for the producer in order to choose that level of employment. The constant expectation locus has a negative or positive slope according to that the $L=L^{\mathrm{d}}\left(s_{t}, h_{t}\right)$ curve intersects the $s=S(L)$ curve from below (i.e. $\left.F^{\prime} /\left(\partial y^{*} / \partial s\right)>S^{\prime}\right)$ or from left (i.e. $\left.F^{\prime} /\left(\partial y^{*} / \partial s\right)<S^{\prime}\right)$. Moreover, even if it is positively sloped, the constant expectation locus is flatter than the constant inventory locus. ${ }^{13}$ To examine the dynamic behavior of $s_{t}$, we differentiate the expectation adjustment equation to obtain

$$
\left.\frac{\partial\left(s_{t+1}-s_{t}\right)}{\partial s}\right|_{s_{t+1}=s_{t}}=\alpha\left(\frac{F^{\prime}}{S^{\prime}} \frac{\partial y^{*}}{\partial s}-S^{\prime}\right)
$$

In either case in which $F^{\prime} /\left(\partial y^{*} / \partial s\right)>S^{\prime}$ or $F^{\prime} /\left(\partial y^{*} / \partial s\right)<S^{\prime}, s_{t+1}<s_{t}$ if $\left(s_{t}, h_{t}\right)$ lies above the $s_{t+1}=s_{t}$ locus.

We plot possible shapes of the constant inventory locus and the constant expectation locus in Fig. 1 in which $F^{\prime} /\left(\partial y^{*} / \partial s\right)>S^{\prime}$ is assumed. An intersection of two loci is an equilibrium state. There are two equilibrium states that we label $\epsilon_{1}$, and $\epsilon_{2}$, respectively. At $\epsilon_{1}$ equilibrium state the lower production, $y_{1}$, takes place (i.e., $y^{*}\left(s_{1}^{*}, h_{1}^{*}\right)=$ $y_{1}$ ) while at $\epsilon_{2}$ equilibrium state the equilibrium production, $y_{2}$, takes place $\left(y^{*}\left(s_{1}^{*}, h_{1}^{*}\right)=y_{1}\right)$. Arrows in Fig. 1 indicate possible movements of trajectories generated by the dynamic system, (16). We can see that $\epsilon_{2}$ equilibrium is a saddle point and hence unstable except one stable path. As is seen below, the stability of $\epsilon_{1}$ equilibrium depends on particular values of the adjustment coefficient, $\alpha$, and of the inventory-expectation ratio, $\beta$.

\footnotetext{
${ }^{13}$ Subtracting (23) from (20) shows that $\left.(\partial h / \partial s)\right|_{h_{t+1}}=h_{t}-\left.(\partial h / \partial s)\right|_{s_{t+1}}=s_{t}=-F^{\prime} /\left(S^{\prime} \partial y^{*} / \partial h\right)>0$.
} 


\subsection{Stability Analysis}

We analyze the local stability at each equilibrium mathematically. The Jacobian matrix, which is obtained by a linear Taylor expansion of the system (16) evaluated at the equilibrium point, is

$$
J=\left(\begin{array}{cc}
1+\alpha\left(\frac{S^{\prime}}{F^{\prime}} \frac{\partial y^{*}}{\partial S}-1\right) & \alpha \frac{S^{\prime}}{F^{\prime}} \frac{\partial y^{*}}{\partial h} \\
\left(1-\frac{S^{\prime}}{F^{\prime}}\right) \frac{\partial y^{*}}{\partial s} & 1+\left(1-\frac{S^{\prime}}{F^{\prime}}\right) \frac{\partial y^{*}}{\partial h}
\end{array}\right)
$$

The determinant, the trace, and the characteristic equation of (24) are, respectively, as follows:

$$
\begin{aligned}
\operatorname{det} J(\alpha)= & (1-\alpha)\left\{1+\left(1-\frac{S^{\prime}}{F^{\prime}}\right) \frac{\partial y^{*}}{\partial h}\right\} \\
& +\alpha \frac{S^{\prime}}{F^{\prime}} \frac{\partial y^{*}}{\partial s} \\
\operatorname{tr} J(\alpha)= & \left\{1+\left(1-\frac{S^{\prime}}{F^{\prime}}\right) \frac{\partial y^{*}}{\partial h}\right\} \\
& +\alpha \frac{S^{\prime}}{F^{\prime}} \frac{\partial y^{*}}{\partial s}+(1-\alpha), \\
\varphi(\lambda)= & \lambda^{2}-\operatorname{tr} J(\alpha) \lambda+\operatorname{det} J(\alpha) .
\end{aligned}
$$

It follows that the characteristic roots are $\lambda_{1,2}=\frac{1}{2} \operatorname{tr} J(\alpha) \pm \sqrt{-D(\alpha)}$ where $D(\alpha)=\operatorname{det} J(\alpha)-$ $\frac{1}{4}(\operatorname{tr} J(\alpha))^{2}$ is the discriminant of the characteristic equation. Depending on the sign of the discriminant and on whether the modulus of the characteristic root is greater or less than unity, the trajectories diverge or converge. Next theorem confirms the graphical intuition that $\epsilon_{2}$ equilibrium is a saddle point.

THEOREM $1 \epsilon_{2}$ equilibrium is a saddle point.

Proof Since $\left(1-S^{\prime} / F^{\prime}\right)<0$ holds at $\epsilon_{2}$ equilibrium by Assumption 2, $-1<\partial y^{*} / \partial h<0$, and 0 $<\partial y^{*} / \partial s$ by Eqs. (9) and (10), then it can be verified that $\varphi(0)=\operatorname{det} J(\alpha)>0$, and $\varphi(1)=-\alpha$ $\left(1-S^{\prime} / F^{\prime}\right)\left(\partial y^{*} / \partial h\right)<0$ for all $\alpha \in(0,1)$. Hence two roots are real and positive. Furthermore one root is greater than unity, and the other is less than unity.
As can be seen in Eq. (22), the slope of the constant expectation locus is either negative or positive according to whether $F^{\prime} /\left(\partial y^{*} / \partial s\right)$ is greater or less than $S^{\prime}$. By Eq. (1), $S^{\prime}=c$, the marginal propensity to consume that is assumed to be constant. Both of $F^{\prime}$ and $\partial y^{*} / \partial s$ depend on the inventory-expectation ratio, $\beta$. To emphasize the dependency of $\partial y^{*} / \partial s$ on the value of $\beta$, we denote $\partial y^{*} / \partial s$ by $f(\beta)$. Returning to Eq. (10), we define two functions of $\beta$ : $\zeta(\beta)=\beta H^{\prime \prime}\left(h+y^{*}\right.$ $(1+\beta) s)$ and $\eta(\beta)=C^{\prime \prime}\left(y^{*}\right)$. Since positive production takes place for $\beta=0, \zeta(0)=0<\eta(0)$. If we assume that these functions intersect only once, say, for $\beta=\beta_{1}$, we then have $f\left(\beta_{1}\right)=1$ and $\zeta^{\prime}\left(\beta_{1}\right)>\eta^{\prime}\left(\beta_{1}\right)$. A derivative of $f(\beta)$ is

$$
\begin{aligned}
f^{\prime}(\beta)= & \left\{\zeta^{\prime}\left(\beta_{1}\right)-\eta^{\prime}(\beta) f(\beta)+(\partial / \partial \beta)\left(H^{\prime \prime}-V^{\prime \prime}\right)\right. \\
& \times[1-f(\beta)]\} /\left\{C^{\prime \prime}+H^{\prime \prime}-V^{\prime \prime}\right\}
\end{aligned}
$$

which leads to $f^{\prime}(\beta)>0$ for $\beta=\beta_{1}$. We assume that this positive relation in the vicinity of $\beta_{1}$ holds globally:

Assumption $3 f^{\prime}(\beta)>0$ for all $\beta \geq 0$, and there is a value of the inventory-expectation ratio, $\beta^{*}$, that satisfies $f\left(\beta^{*}\right)=F^{\prime} / S^{\prime}$, where $F^{\prime}$ and $S^{\prime}$ are evaluated at $\epsilon_{1}$ equilibrium.

Theorem 2 below states that $\epsilon_{1}$ equilibrium is locally stable if a value of the adaptive coefficient, $\alpha$, is confined to an interval, $(0,1)$, and the inventory-expectation ratio, $\beta$, is not so large.

THEOREM 2 For $\beta<\beta^{*}, \epsilon_{1}$ equilibrium is stable.

Proof Since $F^{\prime}>S^{\prime}$ holds at $\epsilon_{1}$ equilibrium by Assumption 2, $\left(F^{\prime} / S^{\prime}\right)\left(\partial y^{*} / \partial s\right)<1$ for $\beta<\beta^{*}$ by Assumption 3, and $-1<\partial y^{*} / \partial h<0$ by Eq. (9), it can be verified that $0<\operatorname{det} J(\alpha)<1$ and $0<$ $\operatorname{tr} J(\alpha)<2$ for all $\alpha$ in an interval, $(0,1)$. Furthermore, $\varphi(0)=\operatorname{det} J(\alpha)>0$ and $\varphi(1)=-\alpha\left(1-S^{\prime} /\right.$ $\left.F^{\prime}\right)\left(\partial y^{*} / \partial h\right)>0$. We then have two cases depending on values of $\alpha$. For $\alpha$ such that $\mathrm{D}(\alpha)<0$, the characteristic roots are real. From the familiar relationship between the roots and coefficients 
of the characteristic equation, $0<\lambda_{1} \lambda_{2}<1,1<$ $\lambda_{1}+\lambda_{2}<2, \varphi(1)=\left(1-\lambda_{1}\right)\left(1-\lambda_{2}\right)>0$. Thus real roots are positive and less than unity so that the trajectories monotonically converge. For $\alpha$ such that $D(\alpha)>0$, the characteristic roots are complex conjugate. Since the modulus of the root is equal to the square root of $\operatorname{det} J(\alpha)$, it is less than unity (i.e., $\bmod (\lambda)=\sqrt{\operatorname{det} J(\alpha)}<1$ ). That is, the trajectories oscillatory converges.

Theorem 3 below shows that the stability of $\epsilon_{1}$, equilibrium may be violated if $\beta$ becomes larger. Before proceeding the instability analysis, we digress to discuss the following two lemmas. These lemmas concern with critical values of the adaptive coefficients, $\alpha_{0}$ and $\alpha_{1}$, for which the dynamics of the system, (16), qualitatively changes.

LEMMA 1 For $\beta \geq \beta^{*}$, there are the adaptive coefficients, $\alpha_{0}$ and $\alpha_{1}$, such that $D\left(\alpha_{0}\right)=0$ and $\operatorname{det} J\left(\alpha_{1}\right)=1$.

Proof We detect the existence of $\alpha_{1}$ first. In the same way as the proof of Theorem 1, it can be verified that $0<\operatorname{det} J(0)<1$ and $\operatorname{det} J(1)>1$. Furthermore, $\operatorname{det} J(\alpha)$ is monotonically increasing for $\beta>\beta^{*}$. Thus, in an interval, $(0,1]$, there is $\alpha_{1}$ that satisfies $\operatorname{det} J\left(\alpha_{1}\right)=1$. Turn to the existence of $\alpha_{0}$. It is also verified that $D(0)=-\frac{1}{4}[\operatorname{det} J(0)-1]^{2}$ $<0$ and $D\left(\alpha_{1}\right)=1-\frac{1}{4}\left[\operatorname{tr} J\left(\alpha_{1}\right]^{2}\right.$ which is positive if $\operatorname{tr} J\left(\alpha_{1}\right)<2$. Substituting $\left(1-\alpha_{1}\right)\left(1-S^{\prime} / F^{\prime}\right)$ $\left(\partial y^{*} / \partial h\right)=\alpha_{1}\left(1-\left(S^{\prime} / F^{\prime}\right)\left(\partial y^{*} / \partial s\right)\right)$, which is obtained from det $J\left(\alpha_{1}\right)=1$, into the second equation of (25), we have $\operatorname{tr} J\left(\alpha_{1}\right)=2+\left(1-\left(S^{\prime} / F^{\prime}\right)\right)$ $\left(\partial y^{*} / \partial h\right) \alpha_{1}$ so that $0<\operatorname{tr} J\left(\alpha_{1}\right)<2$ for $0<\alpha_{1} \leq 1$. Then we have $D\left(\alpha_{1}\right)>0$. Thus there is an adaptive coefficient, $\alpha_{0}$, in an interval, $\left(0, \alpha_{1}\right)$, such that $D\left(\alpha_{0}\right)=0$.

Since $\alpha_{0}$ and $\alpha_{1}$ depend on a value of $\beta$, we denote these by $\alpha_{0}(\beta)$ and $\alpha_{1}(\beta)$, respectively. Lemma 1 implies $\alpha_{0}(\beta)<\alpha_{1}(\beta)$. As used in the proof of Lemma 1, an alternative expression of $\operatorname{det} J\left(\alpha_{1}\right)=1$ is

$$
g\left(\alpha_{1}\right) \equiv \frac{1-\alpha_{1}}{\alpha_{1}}=\frac{1-\left(S^{\prime} / F^{\prime}\right) f(\beta)}{\left(1-S^{\prime} / F^{\prime}\right)\left(\partial y^{*} / \partial h\right)} \equiv \Phi(\beta) .
$$

It can be verified that $g(1)=0, g^{\prime}\left(\alpha_{1}\right)<0$. If we assume $\Phi^{\prime}(\beta)>0,{ }^{14}$ then $\alpha^{\prime}{ }_{1}(\beta)=\Phi^{\prime}(\beta) / g^{\prime}\left(\alpha_{1}\right)$ $<0$. By definition of $\beta^{*}$ in Assumption 3 (i.e., $\left.\left(S^{\prime} / F^{\prime}\right) f\left(\beta^{*}\right)=1\right), \Phi\left(\beta^{*}\right)=0$ and thus $\alpha_{1}\left(\beta^{*}\right)=1$. We summarize these results in

Lemma $2 \alpha_{0}(\beta)<\alpha_{1}(\beta) \leq 1$ for $\beta \geq \beta^{*}$ where equalities hold for $\beta=\beta^{*}$, and $\alpha_{1}^{\prime}(\beta)<0$.

These lemmas imply the following theorem.

THEOREM 3 Given $\beta \geq \beta^{*}, \epsilon_{1}$ equilibrium is locally stable for $0<\alpha<\alpha_{1}(\beta)$ and unstable for $\alpha>\alpha_{1}(\beta)$

Proof For $\alpha>\alpha_{1}(\beta)$, Lemmas 1 and 2 imply $D(\alpha)>0$ and $\operatorname{det} J(\alpha)>1$. The characteristic roots are complex conjugate and their moduli are greater than unity. Thus the trajectories are oscillatory divergence. By the same token, Lemmas 1and 2 imply that $D(\alpha)<0$ and $\operatorname{det} J(\alpha)<1$ for $0<\alpha<\alpha_{0}(\beta)$ and that $D(\alpha)>0$ and $\operatorname{det} J(\alpha)<1$ for $\alpha_{0}(\beta)<\alpha<\alpha_{1}(\beta)$. Thus for $0<\alpha<\alpha_{1}(\beta)$, the real roots are positive and less than unity, and the modulus of the complex root is less than unity. Hence the trajectories monotonically or oscillatory converge to $\epsilon_{1}$ equilibrium.

We turn to the issue of cyclicity in a case where $\epsilon_{1}$ equilibrium is unstable. The following lemma is a truncated version of the Hopf bifurcation theorem. ${ }^{15}$

LeMma 3 Let the mapping $x_{t+1}=G\left(x_{t}, \alpha\right)$, $x_{t} \in R^{2}, \alpha \in R$, have a smooth family of fixed points $x^{*}(\alpha)$ at which the eigenvalues are complex

\footnotetext{
${ }^{14}$ As can be seen in Eq. (9), a sign of $\Phi^{\prime}(\beta)$ depends on the signs of third-order derivatives of the functions involved. If $(\partial / \partial \beta)\left(\partial y^{*} / \partial h\right)$ is negative but its absolute value is small, or positive, we can have $\Phi^{\prime}(\beta)>0$.

${ }^{15}$ See Lorenz (1993, Theorem 3.6, p. 96).
} 


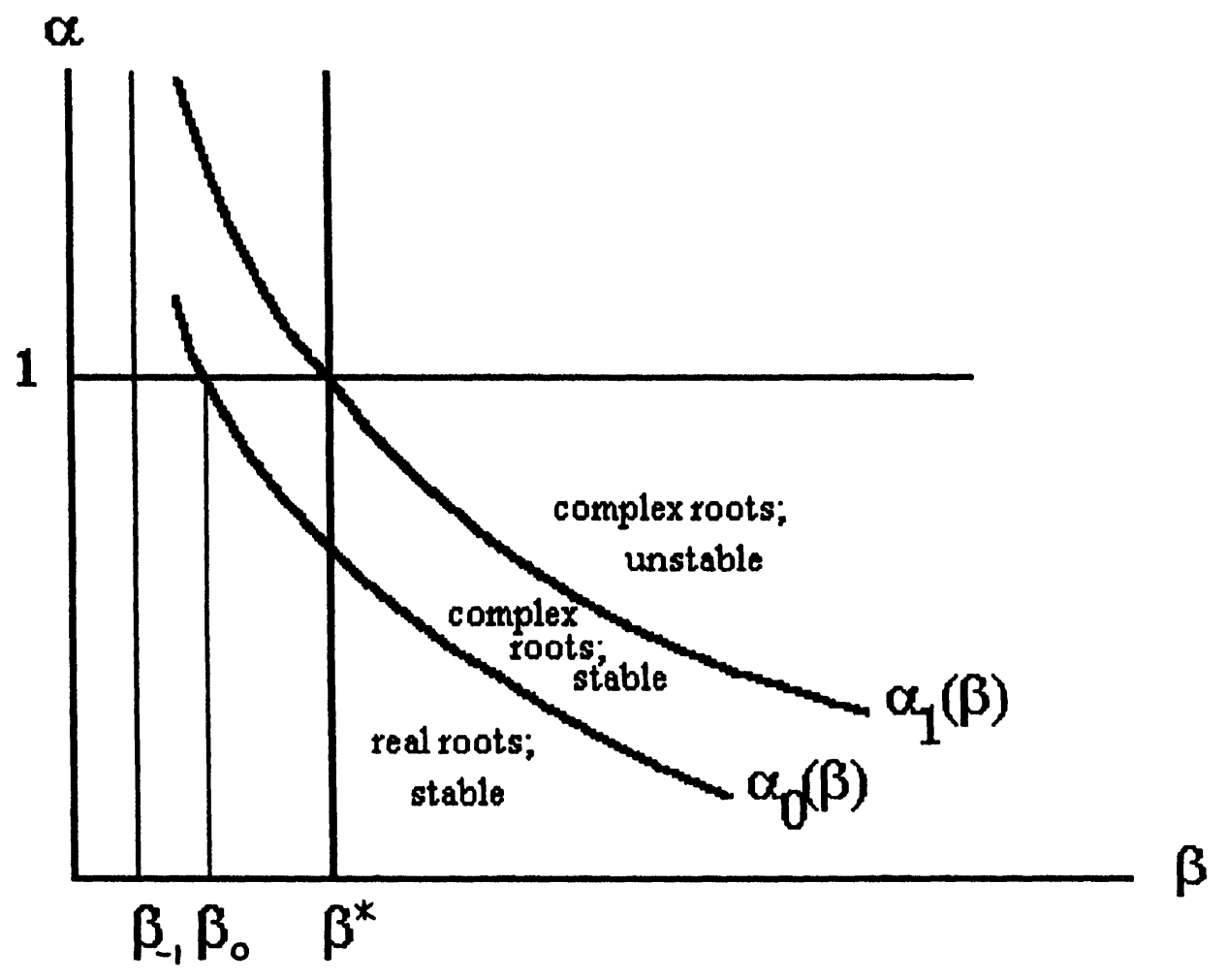

FIGURE 2

conjugate. If there is an $\alpha^{*}$ such that

$\bmod \lambda\left(\alpha^{*}\right)=1$ but $\lambda^{n}\left(\alpha^{*}\right) \neq \pm 1, n=1,2,3,4,5$

and

$$
\frac{\mathrm{d}(\bmod \lambda(\alpha))}{\mathrm{d} \alpha}>0,
$$

then there is an invariant closed curve bifurcating from $\alpha=\alpha^{*}$.

The following theorem guarantees the existence of closed orbits (i.e., endogenous inventory cycles) in the model with adaptive expectations.

THEOREM 4 Given $\beta>\beta^{*}$, there is an invariant closed curve bifurcating from $\alpha=\alpha_{1}(\beta)$.

Proof We first check that $\lambda^{n}\left(\alpha_{1}\right) \neq 1$ for $n=1,2$, $3,4,5{ }^{16}$ Let $\lambda\left(\alpha_{1}\right)=\cos \theta_{1}+i \sin \theta_{1}$ in which $\cos \theta_{1}=\frac{1}{2} \operatorname{tr} J\left(\alpha_{1}\right) . \quad 1<\operatorname{tr} J\left(\alpha_{1}\right)<2$ implies either $\theta_{1} \in(0, \pi / 3)$ or $\theta_{1} \in(-\pi / 3,0)$. Suppose that $\theta_{1}=$ $\pi / n$ for $n>3$. $\lambda^{k}\left(\alpha_{1}\right)=1$ implies $k \theta_{1}=2 \pi r$ for some integer $r$. We can transform the last equation into $k=2 n r>6 r$. This inequality implies $\lambda^{k}\left(\alpha_{1}\right) \neq-1$ for $k<7$. By the same token, $\lambda^{k}\left(\alpha_{1}\right) \neq 1$ for $k<7$. Thus $\lambda^{n}\left(\alpha_{1}\right)$ does not have characteristic roots with absolute values equal to 1 for $n=1,2,3,4,5$. We then verify that the other conditions of Lemma 3 are satisfied. The characteristic roots are complex conjugate for $\alpha \geq \alpha_{1}$ by Lemma 2. Further $\bmod \left(\lambda\left(\alpha_{1}\right)\right)=\sqrt{\operatorname{det} J(\alpha)}=1$ by Lemma 1 and

$$
\begin{aligned}
& \frac{\mathrm{d} \sqrt{\operatorname{det} J(\alpha)}}{\mathrm{d} \alpha} \\
& =\frac{1}{2 \sqrt{\operatorname{det} J(\alpha)}}(\operatorname{det} J(1)-\operatorname{det} J(0))>0,
\end{aligned}
$$

\footnotetext{
${ }^{16}$ We follow the proof of Reichlin (1986, footnote 4, p. 95$)$.
} 
where $\operatorname{det} J(0)<1$ and $\operatorname{det} J(1)>1$ are shown in Lemma 1. The module crosses the unit circle with non-zero speed. This covers the assumptions of the Hopf Theorem. Hence given $\beta>\beta^{*}$, a Hopf bifurcation occurs at $\alpha=\alpha_{1}(\beta)$.

By Eq. (27) and Lemma 2, we can depict a parameter space of the inventory-expectation ratio, $\beta$, and the adaptive coefficient, $\alpha$, as Fig. $2 .{ }^{17}$ $\alpha_{0}(\beta)$ is a boundary between a region for real roots and one for complex roots, and $\alpha_{1}(\beta)$ is a boundary between a stable region and an unstable one.

\subsection{Stability Index}

Taking account of the higher-order terms in the Taylor expansion of the dynamic system, we can compute the stability index of the limit cycle obtained in Theorem 4. To this end, we simplify the model to avoid lengthy calculations and then to make a change of coordinates so that the dynamic system is of the form provided by Wan (1978, see the formulation on p. 168).

AsSumption 4 (1) $F^{\prime \prime}=0$, (2) $k\left(\partial y^{*} / \partial s\right)>1$, where $k=S^{\prime} / F^{\prime}<1$, (3) $k\left(1+\left(\partial y^{*} / \partial s\right) \leq 2\right.$.

Condition (1) assumes a linear production function that consequently implies a linear cost function of production, $C(y)$. We impose conditions (2) and (3) to generate a Hopf bifurcation for admissible values of the adjustment coefficient, $\alpha .{ }^{18} \mathrm{We}$ consider the stability index in the neighborhood of $\epsilon_{1}$ equilibrium, $F^{\prime}>S^{\prime}$ is also assumed. Thus $k=S^{\prime} / F^{\prime}$ is less than unity. Further it is constant by Eq. (1) and Assumption 4(1). Returning to Eqs. (9) and (10), we find by Assumption 4(1) that

$$
\begin{array}{cc}
0<y_{1}^{*}<1+\beta, & y_{2}^{*}=-1, \\
y_{12}^{*}=y_{21}^{*}=y_{22}^{*}=0, & y_{11}^{*} \neq 0,
\end{array}
$$

where $y_{i}^{*}$ is a first-order partial derivative of $y^{*}(s, h)$ with respect to the $i$ th argument, and $y_{i j}^{*}$ is a second-order partial derivative. Taking the Taylor expansion of the dynamic system (16) under Assumption 4, we have

$$
\begin{aligned}
& \left(\begin{array}{c}
s_{t+1} \\
h_{t+1}
\end{array}\right) \\
& =\left(\begin{array}{cc}
-\alpha k & -\alpha k \\
\lambda-\left[1+\alpha\left(k y_{1}^{*}-1\right)\right] & \bar{\lambda}-\left[1+\alpha\left(k y_{1}^{*}-1\right)\right]
\end{array}\right) \\
& \quad \times\left(\begin{array}{c}
s_{t} \\
h_{t}
\end{array}\right)+O^{2},
\end{aligned}
$$

where the higher-order terms up to the third in the Taylor's series are explicitly expressed as

$$
\begin{aligned}
O^{2}= & \frac{1}{2 !}\left(\begin{array}{c}
\alpha k y_{11}^{*} s^{2} \\
(1-k) y_{11}^{*} s^{2}
\end{array}\right) \\
& +\frac{1}{3 !}\left(\begin{array}{c}
\alpha k y_{111}^{*} s^{3} \\
(1-k) y_{111}^{*} s^{3}
\end{array}\right)+O^{4} .
\end{aligned}
$$

We make a change of coordinate so that the system has an appropriate form to compute the stability index. We choose a new coordinate, $(z, \bar{z})$, such that

$$
\begin{aligned}
& \left(\begin{array}{l}
s \\
h
\end{array}\right) \\
& =\left(\begin{array}{cc}
-\alpha k & -\alpha k \\
\lambda-\left[1+\alpha\left(k y_{1}^{*}-1\right)\right] & \bar{\lambda}-\left[1+\alpha\left(k y_{1}^{*}-1\right)\right]
\end{array}\right) \\
& \quad \times\left(\begin{array}{c}
z \\
\bar{z}
\end{array}\right) .
\end{aligned}
$$

In the new coordinate system we have

$\Phi(z)=\lambda z+\frac{(1-\lambda)}{-\alpha k(\bar{\lambda}-\lambda)}\left\{\frac{y_{11} s^{2}}{2}+\frac{y_{11} s^{3}}{6}\right\}+O\left(|z|^{4}\right)$,

\footnotetext{
${ }^{17}$ By Eq. (26), it can be verifed that $\alpha_{1}(\beta)>1$ for $\beta<\beta^{*}$, and as $\beta \rightarrow \beta_{-1}, \lim \alpha_{1}(\beta) \rightarrow \infty$ where $\beta_{-1}$ is defined by $\Phi\left(\beta_{-1}\right)=-1$. By solving $4 \operatorname{det} J(1)=[\operatorname{tr} J(1)]^{2}$, we can detect the existence of the inventory-expectation ratio, $\beta_{1}$, such that $\alpha_{0}\left(\beta_{1}\right)=1$.

${ }^{18}$ It can be verified that $\operatorname{det} J(1) \geq 1$ and $D(1)>0$ under these assumptions.
} 
where $s=-\alpha k(z+\bar{z})$ by Eq. (31). Lengthy computations $^{19}$ show that the stability index, which we denote by $\gamma\left(\alpha_{1}\right)$ (i.e., $f_{\mathrm{b}}(0)$ in Theorem 1 of Wan (1978, p. 168), is reduced to

$\gamma\left(\alpha_{1}\right)=-\frac{(\alpha k)^{2}}{8}\left\{\frac{\operatorname{tr} J\left(\alpha_{1}\right)^{2}+2}{2 D\left(\alpha_{1}\right)} y_{11}^{2}(\alpha k)^{2}-y_{111}\right\}$.

As $D\left(\alpha_{1}\right)>0$, the dynamic system has a repellent invariant cycle (i.e., a subcritical Hopf bifurcation) when $y_{111}<0$.

\section{CONCLUDING REMARKS}

This paper extends the Eckalbar's disequilibrium macro-model. Linear behavioral functions are replaced with non-linear conventional functions that are based on individual's intertemporal optimizing behavior at microeconomic level. The dynamic system consists of the inventory accumulation equation and the adaptively revised expectation adjustment equation. It is demonstrated that the model endowed with a large stock-expectation ratio generates endogenous inventory oscillations. These results suggests that the disequilibrium non-linear dynamics may provide useful explanations for irregularities that are observed in a macro-time series such as those of the real GNP, the unemployment rate, and the inventory investment.

\section{Acknowledgment}

This work was done in part while visiting the University of Southern California. I gratefully acknowledge the hospitality of this institution as well as the financial support from the Fulbright Foundation. I am indebted to Professors Richard Day and Makoto Yano for helpful comments and constructive suggestions. I am also grateful to participants in seminars at University of Southern California and the Savings Economy Research Institute. All remaining errors are my responsibility.

\section{References}

Eckalbar, J. (1985): "Inventory fluctuations in a disequilibrium macro model”, Economic Journal 95, 976-991.

Dana, R. and Malgrange, P. (1984): "The dynamics of a discrete version of a Growth Cycles Model", in: Analyzing the Structure of Econometric Models, Ed. Ancot, J. P., Hague Nijhoff.

Day, R. (1994): Complex Economic Dynamics, Vol. I, MIT press, Cambridge, MA.

Franke, R. and Lux, T. (1993): "Adaptive expectations and perfect foresight in a nonlinear metzlerian model of the inventory cycle", Scandinavian Jouranl of Economics 95, $355-363$.

Honkapohja, S. and Ito, T. (1980): "Inventory dynamics in a simple disequilibrium macroeconomic model", Scandinavian Journal of Economics 82, 184-192.

Lorenz, H.W. (1993): Nonlinear Dynamical Economics and Chaotic Motion, Second, Revised and Enlarged edition, Springer-Verlarg, Berlin.

Matsumoto, A. (1993): "Dynamic complexity in a stochastic rationing model", Journal of Economics 57, 233-259.

Poston, P., Bae, H.O. and Lee, C.N. (1992): "Bifurcation structure of the Eckalbar stock-holding model”, Applied Mathematics and Computation 48, 21-43.

Reichlin, P. (1986): "Equilibrium cycles in an overlapping generations economy with production", Journal of Economic Theory 40, 89-102.

Simonovits, A. (1982): "Buffer stocks and naive expectations in a non-Walrasian dynamic macromodel: stability, cyclicity and chaos", Scandinavian Journal of Economics 84, $571-581$.

Wan, Y.H. (1978): "Computation of the Stability Condition for the Hopf bifurcation of diffeomorphisms on $\mathrm{R}^{2}$, SIAM Journal of Applied Mathematics 34, 167-175.

Zhang, Wei-Bin (1989): "Short-run inventory oscillations in the Eckalbar disequilibrium macro-model", Applied Mathematics and Computation 33, 53-67.

\section{APPENDIX}

In this appendix, we derive the stability index in Eq. (32). An elementary way of Wan (1978) is utilized.

When $C^{\prime \prime}=0$ is assumed in Eqs. (9) and (10), partial derivatives of $y^{*}(s, h)$ (asterisk is omitted for notational simplicity) are as follows:

\footnotetext{
${ }^{19}$ See appendix for full computation.
} 


$$
\left\{\begin{aligned}
y_{1}= & \frac{\partial y^{*}}{\partial s}=\beta \frac{H^{\prime \prime}}{H^{\prime \prime}-V^{\prime \prime}}+1 \\
& \text { that leads to } 0<y_{1}<1+\beta, \\
y_{2}= & \frac{\partial y^{*}}{\partial h}=-\frac{H^{\prime \prime}-V^{\prime \prime}}{H^{\prime \prime}-V^{\prime \prime}}=-1, \\
y_{12}= & y_{21}=\frac{\partial^{2} y^{*}}{\partial s \partial h}=0, \\
y_{22}= & \frac{\partial^{2} y^{*}}{\partial h^{2}}=0 \\
y_{11}= & \frac{\beta}{\left(H^{\prime \prime}-V^{\prime \prime}\right)^{2}}\left\{H^{\prime \prime \prime} V^{\prime \prime}\left(y_{1}-1\right)\right. \\
& \left.-H^{\prime \prime} V^{\prime \prime \prime}\left(y_{1}-(1+\beta)\right)\right\} \neq 0
\end{aligned}\right.
$$

Taking the Taylor expansion of the dynamic system, (16), yields

$$
\Phi\left(\begin{array}{l}
s \\
h
\end{array}\right)=J \cdot\left(\begin{array}{l}
s \\
h
\end{array}\right)+O^{2},
$$

where $J$ is the Jacobian matrix and $O^{2}$ is the higher-order terms. Elements of $J$ are given in Eq. (29) in the text. The determinant and trace of the Jacobian matrix, $J$, are

$$
\begin{aligned}
\operatorname{det} J\left(\alpha_{1}\right) & =k-\alpha_{1} k\left(1-y_{1}\right)=1, \\
\operatorname{tr} J\left(\alpha_{1}\right) & =1+k+\alpha_{1}\left(k y_{1}-1\right) \\
& =2-\alpha_{1}(1-k) \\
(\lambda, \bar{\lambda}) & =A \pm i B
\end{aligned}
$$

where $A=\frac{1}{2} \operatorname{tr} J\left(\alpha_{1}\right)$ and

$$
B=\sqrt{\operatorname{det} J\left(\alpha_{1}\right)-\frac{1}{4}\left(\operatorname{det} J\left(\alpha_{1}\right)\right)^{2}} .
$$

$A^{2}+B^{2}=1$ holds for the bifurcation parameter $\alpha=\alpha_{1}$.

We make a coordinate change by

$$
\left(\begin{array}{l}
s \\
h
\end{array}\right)=\left(\begin{array}{cc}
j_{12} & j_{12} \\
\lambda-j_{11} & \bar{\lambda}-j_{11}
\end{array}\right)\left(\begin{array}{l}
z \\
\bar{z}
\end{array}\right)
$$

where $j_{i k}$ is the $(i, k)$ element of the Jacobian matrix, $J$ (in particular, $j_{12}=-\alpha k$ and $j_{11}=$ $\left.1+\alpha\left(k y_{1}-1\right)\right)$. In this new coordinate, we have

$$
\begin{aligned}
\Phi(z)= & \lambda z+\left(\frac{G_{11}}{2} z^{2}+G_{12} z \bar{z}+\frac{G_{22}}{2} \bar{z}^{2}\right) \\
& +\left(\frac{G_{111}}{6} z^{3}+\frac{G_{112}}{2} z^{2} \bar{z}+\frac{G_{122}}{2} z^{2}+\frac{G_{222}}{6} \bar{z}^{3}\right) \\
& +O^{4}
\end{aligned}
$$

where

$$
\begin{aligned}
& G_{11}=G_{12}=G_{22}=-y_{11}(-\alpha k)^{2} \frac{1-\lambda}{\bar{\lambda}-\lambda}, \\
& G_{112}=y_{111}(-\alpha k)^{2} \frac{1-\lambda}{\bar{\lambda}-\lambda} .
\end{aligned}
$$

According to the Wan's formulation (1978, p. 168 ), the stability index is given by

$$
\begin{aligned}
\gamma\left(\alpha_{1}\right)= & \operatorname{Re}\left[\frac{(1-2 \lambda) \bar{\lambda}^{2}}{2(1-\lambda)} G_{11} G_{12}\right] \\
& +\frac{1}{2} G_{12} \bar{G}_{12}+\frac{1}{4} G_{22} \bar{G}_{22}-\operatorname{Re}\left(\frac{\bar{\lambda} G_{112}}{2}\right) .
\end{aligned}
$$

Using Eqs. (A1), we compute each term in the above formulation, (A2).

$$
\begin{aligned}
& G_{11} G_{12}=\left(\frac{-y_{11}(-\alpha k)^{2}}{2 i B}\right)^{2}(1-\lambda)^{2} \\
&=-\frac{y_{11}^{2}(\alpha k)^{4}}{4 B^{2}}\left\{\left(1-2 A+A^{2}-B^{2}\right)\right. \\
&+i(2 A B-2 B)\} \\
&= \frac{y_{11}^{2}(\alpha k)^{4}}{2 B^{2}}(1-A)(A+i B), \\
& \frac{(1-2 \lambda) \bar{\lambda}^{2}}{2(1-\lambda)}= \frac{(1-2 \lambda)(1-\bar{\lambda}) \bar{\lambda}^{2}}{2(1-\lambda)(1-\bar{\lambda})} \\
&=\frac{(1-A)\left(4 A^{2}-2 A-3\right)+i B\left(4 A^{2}-6 A+1\right)}{4(1-A)},
\end{aligned}
$$




$$
\begin{aligned}
G_{12} \bar{G}_{12} & =G_{22} \bar{G}_{22} \\
& =\left(-y_{11}(-\alpha k)^{2}\right)^{2} \frac{1-\lambda}{\bar{\lambda}-\lambda} \cdot \frac{1-\bar{\lambda}}{\lambda-\bar{\lambda}} \\
& =\frac{y_{11}^{2}(\alpha k)^{4}}{4 B^{2}}(-2 A), \\
\frac{\bar{\lambda}}{2} G_{112} & =\frac{y_{11}(\alpha k)^{2}}{4} \frac{(1-\lambda) \bar{\lambda}}{\bar{\lambda}-\lambda} \\
& =\frac{y_{11}(\alpha k)^{2}}{4}\left(\frac{1}{2}+i \frac{A-1}{2 B}\right) .
\end{aligned}
$$

Substituting the results obtained above, the real parts of complex roots in Eq. (A2) are as follows:

$$
\begin{aligned}
\operatorname{Re}\left[\frac{(1-2 \lambda) \bar{\lambda}^{2}}{2(1-\lambda)} G_{11} G_{12}\right] \\
=\operatorname{Re}\left[\frac { y _ { 1 1 } ^ { 2 } ( \alpha k ) ^ { 4 } } { 8 B ^ { 2 } } \left\{( A + i B ) \left[(1-A)\left(4 A^{2}-2 A-3\right)\right.\right.\right. \\
\left.\left.\left.\quad+i B\left(4 A^{2}-6 A+1\right)\right]\right\}\right] \\
=\frac{y_{11}^{2}(\alpha k)^{4}}{8 B^{2}}(1-A)\left\{A\left(4 A^{2}-2 A-3\right)+(1+A)\right. \\
\left.\quad \times\left(4 A^{2}-6 A+1\right)\right\} \\
=\frac{y_{11}^{2}(\alpha k)^{4}}{8 B^{2}}(1-A)(2 A-1)
\end{aligned}
$$

$$
\operatorname{Re}\left(\frac{\bar{\lambda} G_{112}}{2}\right)=\frac{y_{111}(\alpha k)^{2}}{8}
$$

Hence the stability index, (A2), is

$$
\begin{aligned}
\gamma\left(\alpha_{1}\right)= & \left\{\frac{y_{11}^{2}(\alpha k)^{4}}{8 B^{2}}(1-A)(2 A-1)+\left(\frac{1}{2}+\frac{1}{4}\right)\right. \\
& \left.\times \frac{\left(y_{11}(\alpha k)^{2}\right)^{2}}{4 B^{2}}(-2 A)\right\}-\frac{y_{111}(\alpha k)^{2}}{8} \\
= & -\frac{(\alpha k)^{2}}{8}\left\{\frac{2 A^{2}+1}{B^{2}} y_{11}^{2}(\alpha k)^{2}-y_{111}\right\} .
\end{aligned}
$$

Since $A=\frac{1}{2} \operatorname{tr} J\left(\alpha_{1}\right)$ and $B=\sqrt{D(\alpha)}$, we have Eq. (32) in the text,

$$
\gamma\left(\alpha_{1}\right)=-\frac{(\alpha k)^{2}}{8}\left\{\frac{2\left(\operatorname{tr} J\left(\alpha_{1}\right)\right)^{2}+1}{D\left(\alpha_{1}\right)} y_{11}^{2}(\alpha k)^{2}-y_{111}\right\} .
$$




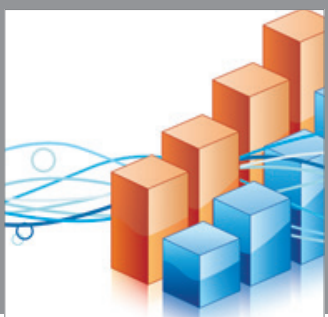

Advances in

Operations Research

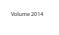

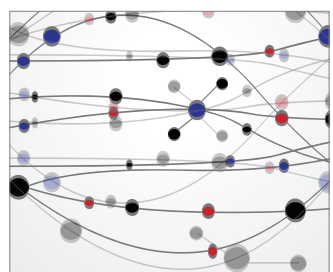

\section{The Scientific} World Journal
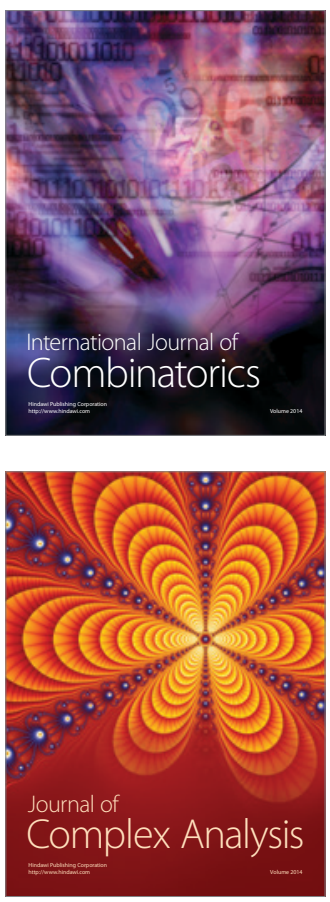

International Journal of

Mathematics and

Mathematical

Sciences
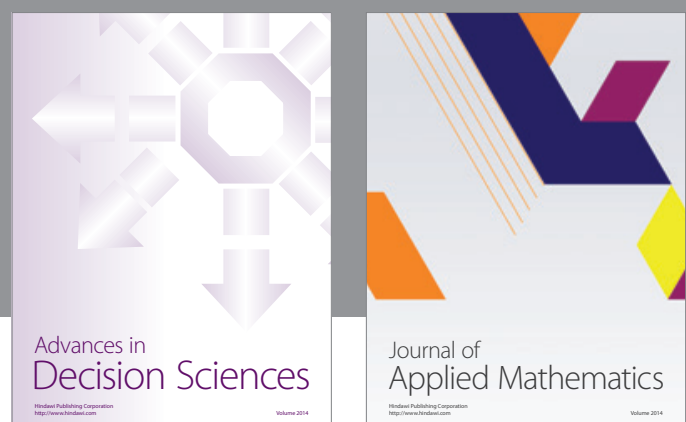

Journal of

Applied Mathematics
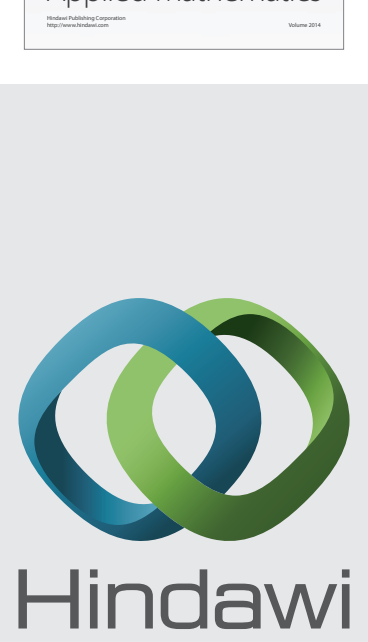

Submit your manuscripts at http://www.hindawi.com
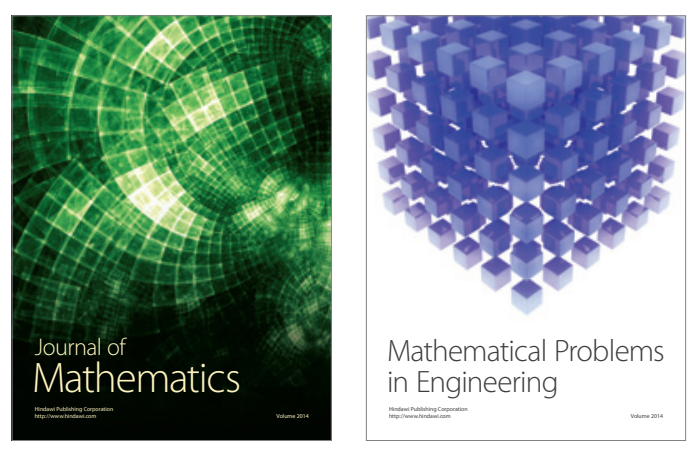

Mathematical Problems in Engineering
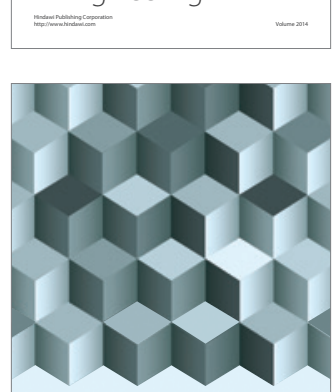

Journal of

Function Spaces
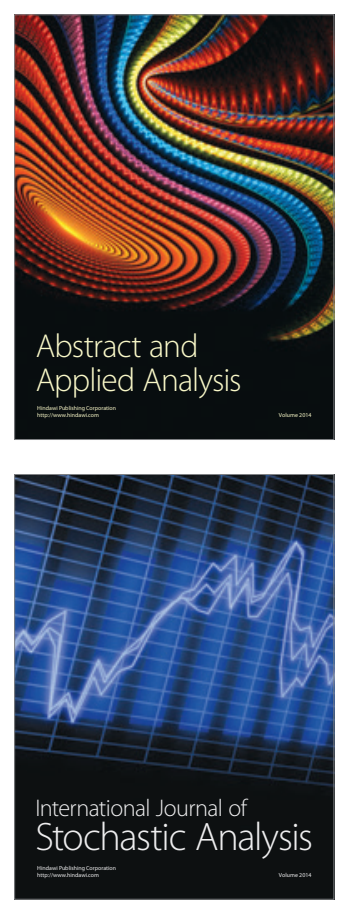

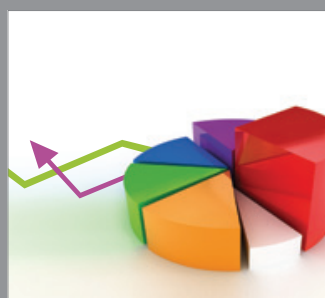

ournal of

Probability and Statistics

Promensencen
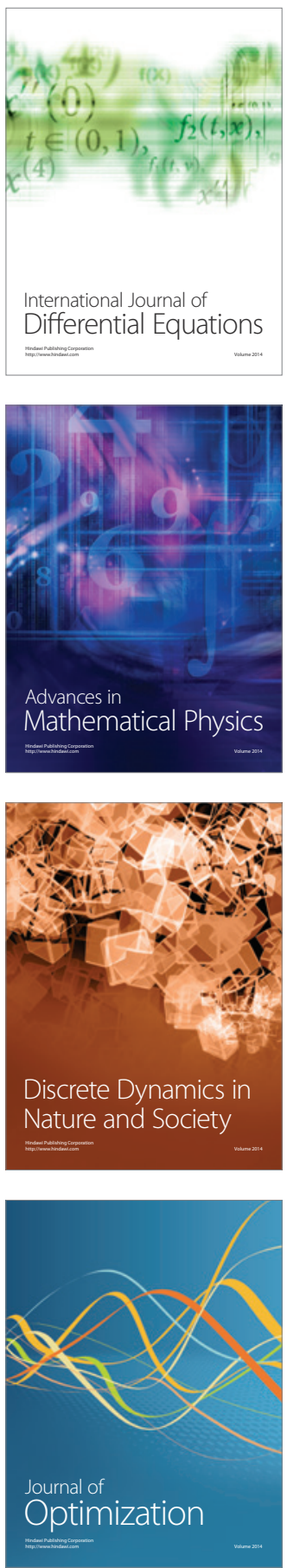